Interfaces and Free Boundaries 16 (2014), 215-241

DOI $10.4171 / \mathrm{IFB} / 318$

\title{
An overdetermined problem with non-constant boundary condition
}

\author{
CHIARA BIANCHINI \\ Dip.to di Matematica “U. Dini”, Università degli Studi di Firenze, Viale Morgagni 67/A, \\ 50134 Firenze, Italy \\ E-mail: cbianchini@math.unifi.it \\ ANTOINE HENROT \\ Institut Elie Cartan, UMR CNRS 7502, Université de Lorraine, \\ Boulevard des Aiguillettes B.P. 70239, 54506 Vandoeuvre-les-Nancy Cedex, France \\ E-mail: antoine.henrot@univ-lorraine.fr \\ PAOLO SALANI \\ Dip.to di Matematica “U. Dini”, Università degli Studi di Firenze, Viale Morgagni 67/A, \\ 50134 Firenze, Italy \\ E-mail:paolo.salani@unifi.it
}

[Received 13 February 2013 and in revised form 29 August 2013]

\begin{abstract}
We investigate an overdetermined torsion problem, with a non-constant positively homogeneous boundary constraint on the gradient. We interpret this problem as the Euler equation of a shape optimization problem, we prove existence and regularity of a solution. Moreover several geometric properties of the solution are shown.
\end{abstract}

2010 Mathematics Subject Classification: Primary 35N25; Secondary: 49Q10, 35B06, 49J20, 49 N60.

Keywords: Torsion problem, overdetermined problem, shape optimization.

\section{Introduction}

An important class of shape optimization problems occurs when a free boundary problem is considered. Of particular interest is the case of overdetermined boundary value problems, which in general corresponds to a classical partial differential equation where both Dirichlet and Neumann boundary conditions are imposed on the boundary of the domain. Obviously this over-determination makes the domain itself unknown. Interesting questions are then the proof of the existence of a solution, possibly uniqueness and the study of qualitative properties of a solution. A very large amount of literature exists for such problems, depending on the governing operator and on the overdetermined conditions which in many cases write as $u=0$ and $|\nabla u|=$ constant on $\partial \Omega$, although several other kinds of overdetermined conditions and operators have been considered in the literature (see for instance [4, 15, 16, 21, 22, 31, 33, 34] and references therein).

In particular here we will deal with a governing operator of the torsion type, that is with the most classical equation:

$$
-\Delta u=1 \quad \text { in } \Omega
$$

and of course everybody knows the famous result by Serrin [32] who proved that if a solution to (1) exists with $u=0$ and $|\nabla u|=$ constant on the boundary of $\Omega$, then the set $\Omega$ must be a ball 
and the function $u$ is radial. After Serrin, many authors investigated such a problem and stability results hold. More precisely it has been proved in [3] and in [7] that if $|\nabla u|$ is almost constant on the boundary of $\Omega$, then $\Omega$ is not far from being a ball. A natural question is then to investigate what happens in the case of a "genuine" non-constant boundary condition for the gradient. In particular here we are interested in an overdetermined condition of the type

$$
|\nabla u(x)|=g(x) \quad \text { on } \partial \Omega .
$$

Let us quote that the same conditions have been already considered for differential problems of the torsion and of the Bernoulli types in $[1,2,5,19,24]$. On the other hand in [6] the Bernoulli interior problem equipped with overdetermined condition written as $|\nabla u(x)|=g(v(x))$ is considered, where $v$ is the outer unit normal of the free boundary. At our knowledge an analogous overdetermined condition (which would be very natural) for the torsion problem has not been considered yet.

Let us now describe in detail the problem we are interested in; we will relate it below to the existing literature.

For any bounded open set $\Omega$ (or set of finite measure) we denote by $u_{\Omega}$ the stress function of $\Omega$; that is the solution of the torsion problem:

$$
\begin{cases}-\Delta u_{\Omega}=1 & \text { in } \Omega \\ u_{\Omega}=0 & \text { on } \partial \Omega\end{cases}
$$

or of its weak form

$$
u_{\Omega} \in H_{0}^{1}(\Omega), \quad \forall v \in H_{0}^{1}(\Omega): \int_{\Omega} \nabla u_{\Omega} \nabla v=\int_{\Omega} u_{\Omega} v,
$$

where $H^{1}(\Omega)$ is the Sobolev space of functions in $L^{2}(\Omega)$ whose first derivatives are in $L^{2}(\Omega)$ and $H_{0}^{1}(\Omega)$ is the closure in $H^{1}(\Omega)$ of smooth functions compactly supported in $\Omega$. Notice that the stress function $u_{\Omega}$ can be characterized as

$$
\begin{aligned}
G_{\Omega}\left(u_{\Omega}\right) & =\min \left\{G_{\Omega}(v), v \in H_{0}^{1}(\Omega)\right\} \text { where } \\
G_{\Omega}(v) & =\frac{1}{2} \int_{\Omega}|\nabla v|^{2} d x-\int_{\Omega} v d x .
\end{aligned}
$$

Let $g$ be a function defined on $\mathbb{R}^{N}$ and satisfying

$$
\left\{\begin{array}{l}
g: \mathbb{R}^{N} \rightarrow \mathbb{R} \text { positively homogeneous of degree } \alpha \\
\quad \text { (i.e. } g(t x)=t^{\alpha} g(x) \forall t>0, \forall x \in \mathbb{R}^{N}, \alpha>0 \text { ), } \\
g \text { Hölder continuous, } g>0 \text { outside } 0 .
\end{array}\right.
$$

We are interested in solving the following overdetermined free boundary problem of the torsional type with a non constant boundary condition:

$$
\begin{cases}-\Delta u_{\Omega}=1 & \text { in } \Omega \\ u_{\Omega}=0 & \text { on } \partial \Omega \\ \left|\nabla u_{\Omega}\right|=g(x) & \text { on } \partial \Omega\end{cases}
$$


In this context, this problem is close to the one considered by B. Gustafsson and H. Shahgholian in [19]. In fact they study the partial differential equation $-\Delta u=f$ where $f$ is a function (or a measure) whose positive part $(f)^{+}$has compact support (here and later we will denote by $(f)^{+}$ the positive part of the function $f$, that is $\max \{0, f\}$ ). This makes a real difference with (6) as will be clear in a while. Indeed they use the Alt-Caffarelli approach, consisting in minimizing

$$
G_{\Omega}\left(u_{\Omega}\right)+\frac{1}{2} \phi(\Omega)
$$

where

$$
\phi(\Omega):=\int_{\Omega} g^{2}(x) d x
$$

(they write it as a problem in the calculus of variations replacing $\Omega$ by $\{u>0\}$ but it does not change anything). Unfortunately, in our case, the fact that the support of $(f)^{+} \equiv 1$ is the whole $\mathbb{R}^{N}$ makes the minimization problem (7) in general ill-posed, since the infimum can be $-\infty$ as it is easily seen by explicit computations in the radial case. This is the reason for we have chosen a different method.

We use a variational approach which consists in looking at (6) as the optimality conditions of some shape optimization problem. More precisely, let $J$ be the functional defined as the opposite of the torsional rigidity:

$$
J(\Omega)=\frac{1}{2} \int_{\Omega}\left|\nabla u_{\Omega}\right|^{2} d x-\int_{\Omega} u_{\Omega} d x=-\frac{1}{2} \int_{\Omega} u_{\Omega} d x=-\frac{1}{2} \int_{\Omega}\left|\nabla u_{\Omega}\right|^{2} d x .
$$

and consider $\phi$ as defined in (8). The shape optimization problem we are interested in, consists in minimizing $J$ with the constraint

$$
\phi(\Omega):=\int_{\Omega} g^{2}(x) d x \leqslant 1 .
$$

Let us point out that this introduces a further difficulty since we have to deal with a Lagrange multiplier. The choice of a homogeneous function $g$, allows us to encounter this difficulty (see the proof of Corollary 2.2) since it permits to estimate the value of the Lagrange multiplier. However we point out that the existence of a solution to the shape optimization problem is guaranteed under the simple assumption $g(x)>0$ outside the origin and $\lim g(x)=+\infty$ for $|x| \rightarrow+\infty$.

We remark that this shape optimization problem is a variant of the famous Saint-Venant problem, and hence it has its own practical interest. In the classical Saint-Venant problem one looks for the shape of the set with given area which has maximal torsional rigidity; in [29] G. Polyà proved that the answer is the ball. Here we solve the same problem in the class of non-uniformly dense sets, whose density depends on the function $g$.

The paper is organized as follows. In Section 2 we prove the main results of the paper: existence and regularity of a minimizer for our shape optimization problem and, as a consequence, the existence of a solution to the free boundary problem. In Section 3 we prove some basic properties: the origin is in general inside the solution, monotonicity with respect to $g$ and uniqueness of the solution when $\alpha>1$. In Section 4 we investigate starshape and we prove the starshapedness of solutions for $\alpha \neq 1$. Section 5 is devoted to prove the convexity of the solution, under suitable assumptions. In Section 6 we prove some symmetry results and we study the stability of the radiality when $g$ is close to be radially symmetric. Finally in Section 7 we investigate the relationship between the shape of the solution and the shape of the level sets of $g$, giving some a priori bounds for the solution $\Omega$ in terms on $G_{1}=\left\{x \in \mathbb{R}^{n}: g(x) \leqslant 1\right\}$. 


\section{The shape optimization problem}

We consider the energy functional $J(\cdot)$ defined in (9). We recall that, by the maximum principle, $J$ is decreasing with respect to set inclusion that is: $\Omega_{1} \subset \Omega_{2}$ implies $J\left(\Omega_{1}\right) \geqslant J\left(\Omega_{2}\right)$.

In this section, we want to minimize the functional $J(\Omega)$ among open sets satisfying (10). Let us remark that the measure of sets $\Omega$ satisfying (10) is bounded since $g(x)$ diverges at infinity for homogeneity.

As already emphasized, the homogeneity of $g$ plays a crucial role. In particular it makes the problem having a nice behavior with respect to homotheties. More precisely, for every $t>0$, $\Omega \subset \mathbb{R}^{N}$, it holds

$$
\begin{aligned}
& J(t \Omega)=-\frac{1}{2} \int_{t \Omega} t^{2} u_{\Omega}(x / t) d x=t^{2+N} J(\Omega), \\
& \phi(t \Omega)=\int_{t \Omega} g^{2}(x) d x=t^{2 \alpha+N} \phi(\Omega),
\end{aligned}
$$

where the first equality follows from the fact that the stress function of $t \Omega$ is

$$
u_{t \Omega}(x)=t^{2} u_{\Omega}\left(\frac{x}{t}\right) .
$$

Therefore the two following problems are equivalent from a qualitative point of view:

$$
\begin{gathered}
\min _{\Omega \subset \mathbb{R}^{N}}\{J(\Omega): \phi(\Omega) \leqslant 1\}, \\
\min _{\Omega \subset \mathbb{R}^{N}} \phi(\Omega)^{-\frac{2+N}{2 \alpha+N}} J(\Omega) .
\end{gathered}
$$

Let us state our main result on existence and regularity of a solution to Problem (11) or (12), whose proof is given in Section 2.1. In particular we stress that the existence part in Theorem 2.1 does not need the homogeneity property of $g$ but it holds under the weaker assumption $g>0$ outside the origin and $g$ tends to infinity for $|x| \rightarrow \infty$.

Theorem 2.1 Problem (11) admits a solution $\Omega$. This one is $C^{1, \beta}$ in dimension $N=2$. In dimension $N \geqslant 3$, the reduced boundary $\partial_{\text {red }} \Omega$ is $C^{1, \beta}$ and $\partial \Omega \backslash \partial_{\text {red }} \Omega$ has zero (N-1)-Hausdorff measure.

The existence of a solution to the overdetermined Free Boundary Problem (6) follows:

COROllary 2.2 Let $g$ satisfy (5) for some $\alpha>0, \alpha \neq 1$. Then there exists a solution to the overdetermined Free Boundary Problem (6).

REMARK 2.3 The overdetermined boundary condition $\left|\nabla u_{\Omega}(x)\right|=g(x)$ holds on the regular part of the boundary of $\Omega$.

Proof of Corollary 2.2. Let $\Omega$ be a solution of the shape optimization problem (11) given by Theorem 2.1. Since the reduced boundary of $\Omega$ is $C^{1, \beta}$, by classical regularity results, the gradient of $u_{\Omega}$ is defined almost everywhere on the boundary. We can then write the optimality conditions. For that purpose, we use the classical shape derivative as defined, for example in [23, chapter 5]. The derivative of the functional $J$ at $\Omega$ in the direction of some deformation field $V$ is given by

$$
d J(\Omega ; V)=-\int_{\Omega}\left\langle\nabla u_{\Omega}, \nabla u^{\prime}\right\rangle-\frac{1}{2} \int_{\partial \Omega}\left|\nabla u_{\Omega}\right|^{2}\langle V, n\rangle,
$$


where $u^{\prime}$ is the derivative with respect to the domain of $u_{\Omega}$, solution of

$$
\begin{cases}-\Delta u^{\prime}=0 & \text { in } \Omega \\ u^{\prime}=-\frac{\partial u_{\Omega}}{\partial n}\langle V, n\rangle & \text { on } \partial \Omega .\end{cases}
$$

By Green formula and (14), $\int_{\Omega}\left\langle\nabla u_{\Omega}, \nabla u^{\prime}\right\rangle=0$. On the other hand, the derivative of the constraint $\phi$ is

$$
d \phi(\Omega ; V)=\int_{\partial \Omega} g^{2}\langle V, n\rangle
$$

By the optimality condition there exists a Lagrange multiplier $\mu$ such that, for any deformation field $V$, we have

$$
d J(\Omega ; V)=\mu d \phi(\Omega ; V),
$$

and, according to (13), (15) this writes as

$$
-\frac{1}{2} \int_{\partial \Omega}\left|\nabla u_{\Omega}\right|^{2}\langle V, n\rangle=\mu \int_{\partial \Omega} g^{2}\langle V, n\rangle
$$

Since equality (16) must hold for any $V$, we get

$$
\left|\nabla u_{\Omega}\right|^{2}=-2 \mu g^{2} \quad \text { on } \partial \Omega \text {. }
$$

Let us remark that $\mu$ cannot be zero by unique continuation property (or Hopf's lemma). Now, replacing $\Omega$ by $t \Omega$ where

$$
t=(-2 \mu)^{\frac{1}{2(\alpha-1)}},
$$

and taking into account that

$$
\left|\nabla u_{t \Omega}(x)\right|=t\left|\nabla u_{\Omega}(x / t)\right|=t(-2 \mu)^{1 / 2} g(x / t)=t^{1-\alpha}(-2 \mu)^{1 / 2} g(x)=g(x),
$$

we get the desired result.

REMARK 2.4 The case $\alpha=1$ is a special one. As we can see explicitly in the radially symmetric situation, it is possible to have no solution or an infinite number of solutions. Indeed, let $g(x)=$ $a|x|$, as it is easily proved by Schwarz symmetrization (see section 6), the solution has to be a ball. Now, looking for a ball $B_{R}$ of radius $R$ solving (6) is equivalent to solve $g(R)=R / N$ (because $\left.u_{B_{R}}=\left(R^{2}-|x|^{2}\right) / 2 N\right)$ and the result follows according to the value of $a$.

\subsection{Proof of Theorem 2.1}

The proof splits into two parts which are separated in four paragraphs. In the first part we prove the existence (and boundedness) of a solution, while in the latter the proof of regularity is presented.

More precisely in Paragraph 2.1.1 we follow the lines of [18], cf. also [23] and we use a concentration-compactness argument as in [10] to prove the existence of a minimizer which is a quasi-open set and which may be unbounded. We refer to [23] for a precise definition and discussion of the concept of quasi-open set; let us only remind that if $u \in H^{1}\left(\mathbb{R}^{N}\right)$, then its super level sets $\{u>t\}$ are quasi-open, for each $t \in \mathbb{R}$.

In Paragraph 2.1.2, using the notion of local shape subsolution introduced in [11], we prove that the minimizer is in fact bounded. In Paragraphs 2.1.4, 2.1.3 we prove the regularity of the minimizer as in [8] (see also [9]). The main difficulty is to prove that it is actually an open set, then we can conclude to higher regularity by classical techniques from free boundary problems like in [5] and [19]. 
2.1.1 Proof of existence. Let us introduce the following auxiliary problem

$$
\min \left\{G(v) ; v \in H^{1}\left(\mathbb{R}^{N}\right), \phi\left(\Omega_{v}\right) \leqslant 1\right\},
$$

where $\Omega_{v}$ denotes the quasi-open set $\Omega_{v}:=\left\{x \in \mathbb{R}^{N} ; v(x)>0\right\}, \phi$ is defined in (8) and $G$ is the functional defined by

$$
G(v)=\frac{1}{2} \int_{\mathbb{R}^{N}}|\nabla v|^{2} d x-\int_{\mathbb{R}^{N}} v d x .
$$

Let us prove the existence of a minimizer $u$ for problem (17).

We use the classical Poincare inequality, valid for any set of bounded measure (see Lemma 4.5.3 in [23]). As already noticed, the constraint $\phi\left(\Omega_{v}\right) \leqslant 1$ implies that the measure of $\Omega_{v}$ is uniformly bounded, that is $\exists m>0,\left|\Omega_{v}\right| \leqslant m$. The Poincaré inequality writes as follows: there exists $C=C(N)>0$ such that for every $v \in H^{1}\left(\mathbb{R}^{N}\right)$ satisfying $\left|\Omega_{v}\right| \leqslant m$ it holds

$$
\int_{\mathbb{R}^{N}} v^{2} \leqslant C m^{\frac{2}{N}} \int_{\mathbb{R}^{N}}|\nabla v|^{2} .
$$

Therefore, since $\int_{\Omega_{v}} v \leqslant m^{1 / 2}\left(\int_{\mathbb{R}^{N}} v^{2}\right)^{1 / 2}$, we have

$$
2 G(v) \geqslant \int_{\mathbb{R}^{N}}|\nabla v|^{2}-C^{\prime}\|v\|_{H^{1}\left(\mathbb{R}^{N}\right)},
$$

and $G(v)$ is estimated from below and a minimizing sequence $u_{n}$ is necessarily bounded in $H^{1}\left(\mathbb{R}^{N}\right)$.

Now we use a concentration compactness argument for the quasi-open sets $A_{n}=\left\{u_{n}>0\right\}$. Following [10, Theorem 2.2] two situations may occur:

- Dichotomy: There exists a sequence $\tilde{A}_{n} \subseteq A_{n}$ such that $\tilde{A}_{n}=A_{n}^{1} \cup A_{n}^{2}$ with $d\left(A_{n}^{1}, A_{n}^{2}\right) \rightarrow+\infty$ and $\lim \inf \left|A_{n}^{i}\right|>0$. The resolvent operators satisfy $\left\|R_{A_{n}}-R_{A_{n}^{1} \cup A_{n}^{2}}\right\| \rightarrow 0$ in the operator norm (see [10] for details on the resolvent operator).

- Compactness: There exists a sequence of vectors $y_{n} \in \mathbb{R}^{N}$ and a positive Borel measure $\mu$ (vanishing on sets of zero capacity) such that $y_{n}+A_{n} \gamma$-converges to the measure $\mu$ (and the resolvent operators satisfy $\left\|R_{y_{n}+A_{n}}-R_{\mu}\right\| \rightarrow 0$ in the operator norm).

Notice that in the situation of Problem (17), dichotomy cannot occur because since $g^{2} \rightarrow+\infty$ at infinity, the constraint $\int_{A_{n}} g^{2} \leqslant 1$ prevents a subpart of $A_{n}$ of measure bounded from below to move to infinity. Thus, we are in the compactness situation and we denote by $A_{\mu}$ the regular set of the limit measure $\mu$, defined as

$$
A_{\mu}:=\{\bigcup A: A \text { is finely open, } \mu(A)<\infty\} .
$$

Then the sequence $v_{n}(x)=u_{n}\left(x-y_{n}\right)=R_{y_{n}+A_{n}}(1)$ converges to $v=R_{\mu}(1) \in H_{0}^{1}\left(A_{\mu}\right)$ weakly in $H^{1}$ and almost everywhere (and $A_{\mu}=\{v>0\}=\Omega_{v}$ ). Notice that at this point we can not say that $\Omega_{v}$ provides a solution to minimization problem (17), since the constraint is not translation invariant. We can avoid this problem arguing for instance as in [12], proving that the sequence $\left\{y_{n}\right\}$ is bounded, thanks again to the behavior of $g$ at infinity. Indeed first choose $R>0$ such that $\int_{B(O, R)} v^{2} d x=\alpha>0$ (here and in the sequel we denote by $B(x, R)$ the ball centered at $x$ with radius $R$ ); by the convergence of $v_{n}$ to $v$, we have

$$
\int_{B(0, R)} v_{n}^{2} d x=\int_{B\left(-y_{n}, R\right)} u_{n}^{2} d x=\int_{B\left(-y_{n}, R\right) \cap A_{n}} u_{n}^{2} d x \geqslant \frac{\alpha}{2}
$$


for $n$ large enough. Since $\left|A_{n}\right|$ is bounded, $\left\|u_{n}\right\|_{\infty}$ is bounded, say $\left\|u_{n}\right\|_{\infty} \leqslant M$; then (20) implies

$$
\left|A_{n} \cap B\left(-y_{n}, R\right)\right| \geqslant \frac{\alpha}{2 M^{2}},
$$

whence

$$
1 \geqslant \int_{A_{n}} g^{2} d x \geqslant \int_{A_{n} \cap B\left(-y_{n}, R\right)} g^{2} d x \geqslant \frac{\alpha}{2 M^{2}} \inf _{B\left(-y_{n}, R\right)} g^{2} .
$$

The latter leads to a contradiction if we assume that $\left\|y_{n}\right\|$ is unbounded, since we would have $\inf _{B\left(-y_{n}, R\right)} g^{2} \rightarrow \infty$. So far, we have proved $y_{n}$ is bounded. Then it converges to some $y_{0}$ (up to a subsequence) and the sequence $u_{n}$ converges to $u(x)=v\left(x+y_{0}\right)$ weakly in $H^{1}$ and almost everywhere. We set $\Omega^{*}=\{u>0\}=A_{\mu}-y_{0}$ and by Fatou Lemma, we infer that

$$
\int \chi \Omega_{\Omega^{*}} g^{2} d x \leqslant \liminf \int \chi_{\Omega_{u_{n}}} g^{2} d x \leqslant 1
$$

and so the constraint is satisfied. We deduce that $\Omega^{*}$ provides a solution of the shape optimization problem (9), (10), as it is classical in situations where the objective function $J$ is monotone decreasing with respect to set inclusion.

2.1.2 Proof of boundedness. We recall the definition of local shape subsolution introduced by D. Bucur in [11]: a set $A$ is a local shape subsolution for the energy problem if there exist $\delta>0$ and $\Lambda>0$ such that for any quasi-open set $\tilde{A} \subset A$ with $\left\|u_{\tilde{A}}-u_{A}\right\|_{L^{2}}<\delta$ we have

$$
J(A)+\Lambda|A| \leqslant J(\tilde{A})+\Lambda|\tilde{A}| .
$$

In [11, Theorem 2.2], it is proved that any local shape subsolution is bounded (and has finite perimeter). Thus our aim is to prove that $\Omega^{*}$ is a local shape subsolution. We argue by contradiction: let us assume that there exists a sequence $\lambda_{n}$ going to 0 and a sequence $\Omega_{n} \subset \Omega^{*}$ such that

$$
J\left(\Omega_{n}\right)+\lambda_{n}\left|\Omega_{n}\right|<J\left(\Omega^{*}\right)+\lambda_{n}\left|\Omega^{*}\right| .
$$

We can assume that $\Omega_{n}$ is an increasing sequence converging to $\Omega^{*}$ in the $L^{2}\left(\mathbb{R}^{N}\right)$-norm of $u_{\Omega_{n}}$, that is $\left\|u_{n}-u\right\|_{L^{2}\left(\mathbb{R}^{N}\right)}$ tends to zero, where $u=u_{\Omega^{*}}$. Indeed, if $\Omega_{n}$ converges to a strictly smaller set, then equation (22) cannot hold by monotonicity of the energy $J$ and this would give a contradiction. Fix $t_{n}>1$ such that

$$
\phi\left(t_{n} \Omega_{n}\right)=t_{n}^{2 \alpha+N} \int_{\Omega_{n}} g^{2}=1 .
$$

Then necessarily $t_{n} \rightarrow 1$. By minimality of $\Omega^{*}, J\left(t_{n} \Omega_{n}\right)=t_{n}^{N+2} J\left(\Omega_{n}\right) \geqslant J\left(\Omega^{*}\right)$.

Plugging into (22) yields

$$
J\left(\Omega^{*}\right)\left[\frac{1-t_{n}^{N+2}}{t_{n}^{N+2}}\right] \leqslant \lambda_{n}\left(\left|\Omega^{*}\right|-\left|\Omega_{n}\right|\right) .
$$

We divide both sides of (23) by $t_{n}^{N+2 \alpha}-1=\int_{\Omega^{*}} g^{2}\left(\int_{\Omega_{n}} g^{2}\right)^{-1}-1$ and we get

$$
\frac{t_{n}^{N+2}-1}{t_{n}^{N+2 \alpha}-1}\left(-\frac{J\left(\Omega^{*}\right)}{t_{n}^{N+2}}\right) \leqslant \frac{\lambda_{n}\left|\Omega^{*} \backslash \Omega_{n}\right| \int_{\Omega_{n}} g^{2}}{\int_{\Omega^{*} \backslash \Omega_{n}} g^{2}} .
$$


Now obviously, we just need to prove boundedness far from 0. Actually the proof of boundedness of shape subsolution in Bucur's paper [11] is done by contradiction and consists in applying an estimate of Alt-Caffarelli type (which follows from the definition of shape subsolution) to a sequence of points $x_{n} \in \Omega$ whose norm is going to $+\infty$. Therefore we can assume that we are outside a fixed ball $B(0, R)$ and

$$
\frac{\int_{\Omega^{*} \backslash \Omega_{n}} g^{2}}{\left|\Omega^{*} \backslash \Omega_{n}\right|} \geqslant \min _{\mathbb{R}^{N} \backslash B(0, R)} g^{2}>0 .
$$

We pass to the limit $\lambda_{n} \rightarrow 0$ and $t_{n} \rightarrow 1$; since the left-hand side of (24) converges to $\frac{N+2}{N+2 \alpha}\left(-J\left(\Omega^{*}\right)\right)>0$ and the right-hand side tends to 0 , we reach the desired contradiction.

2.1.3 Proof of regularity. The proof of regularity of the optimal shape is very similar to the proof given in [8] (see also [9]) where the constraint is $\left|\Omega_{u}\right| \leqslant 1$ instead of $\int_{\Omega_{u}} g^{2} \leqslant 1$. These papers are themselves inspired by [5] and [19]. Thus, in the sequel, we will mainly emphasize the particularities of our situation.

Before giving the details, we need to show some preliminary results.

Let us denote by $u$ the minimizer of problem (17). Since we know that the minimizer is bounded, let us denote by $D$ a fixed ball containing $\Omega_{u}$. The first step is to prove that $u$ is continuous in $D$ (and therefore the set $\Omega_{u}=\{x: u(x)>0\}$ is open). To get rid of the constraint (in order to be able to test with a wider class of functions), we first prove that the minimization problem (17) is equivalent to a penalized problem.

Lemma 2.5 There exists $\lambda>0$ such that for any $v \in H_{0}^{1}(D)$

$$
G(u) \leqslant G(v)+\lambda\left(\int_{\Omega_{v}} g^{2}(x) d x-1\right)^{+} .
$$

It is remarkable that the two problems are equivalent, not only when $\lambda$ goes to infinity as usual, but for a finite value of $\lambda$.

Proof of Lemma 2.5. For a fixed $\lambda>0$, let us denote by $G_{\lambda}$ the functional

$$
G_{\lambda}(v):=G(v)+\lambda\left(\phi\left(\Omega_{v}\right)-1\right)^{+} .
$$

The existence of a minimizer $u_{\lambda}$ for the problem

$$
\inf _{v \in H_{0}^{1}(D)} G_{\lambda}(v),
$$

is obtained in an analogous way as the existence of a minimizer for problem (17) above. If $\phi\left(\Omega_{u_{\lambda}}\right) \leqslant 1$, we get $G_{\lambda}\left(u_{\lambda}\right)=G\left(u_{\lambda}\right)$ and since $u_{\lambda}$ and $u$ are both minimizers of $G_{\lambda}$ and $G$, the result follows. It remains to prove that we cannot have

$$
\phi\left(\Omega_{u_{\lambda}}\right)>1,
$$

for $\lambda$ large enough. Assume, by contradiction, that it is the case and let us introduce $u^{t}=\left(u_{\lambda}-t\right)^{+}$. Differentiating with respect to $t$ and using the co-area formula, we get

$$
\begin{array}{r}
\left.\frac{d}{d t}\right|_{t=0} \int_{\left\{u_{\lambda}>t\right\}}\left|\nabla u_{\lambda}\right|^{2} d x=-\int_{\left\{u_{\lambda}=t\right\}}\left|\nabla u_{\lambda}\right| d \mathcal{H}^{N-1}, \\
\left.\frac{d}{d t}\right|_{t=0} \int_{\left\{u_{\lambda}>t\right\}} g^{2} d x=-\int_{\left\{u_{\lambda}=t\right\}} \frac{g^{2}}{\left|\nabla u_{\lambda}\right|} d \mathcal{H}^{N-1},
\end{array}
$$


while

$$
\left.\frac{d}{d t}\right|_{t=0} \int_{\left\{u_{\lambda}>t\right\}}\left(u_{\lambda}-t\right) d x=-\int_{\left\{u_{\lambda}>t\right\}} d x .
$$

Thus $\left.\frac{d}{d t}\right|_{t=0} G_{\lambda}\left(u^{t}\right) \geqslant 0$ yields

$$
\int_{\left\{u_{\lambda}=t\right\}} \frac{1}{2}\left|\nabla u_{\lambda}\right|+\lambda \frac{g^{2}}{\left|\nabla u_{\lambda}\right|} d \mathcal{H}^{N-1} \leqslant \int_{\left\{u_{\lambda}>t\right\}} d x \leqslant|D|
$$

Now

$$
\int_{\left\{u_{\lambda}=t\right\}} \frac{1}{2}\left|\nabla u_{\lambda}\right|+\lambda \frac{g^{2}}{\left|\nabla u_{\lambda}\right|} d \mathcal{H}^{N-1} \geqslant \sqrt{2 \lambda} \int_{\left\{u_{\lambda}=t\right\}} g d \mathcal{H}^{N-1},
$$

therefore, if we can prove that $\int_{u_{\lambda}=t} g d \mathcal{H}^{N-1}$ is estimated from below by a positive constant, (27) would lead to the desired contradiction for $\lambda$ large enough. This is the content of the following lemma.

Lemma 2.6 There exists a positive constant $C$ such that for any measurable set $\omega \subset D$ with $\int_{\omega} g^{2} d x \geqslant 1$, it holds

$$
\int_{\partial \omega} g d \mathcal{H}^{N-1} \geqslant C
$$

Proof. Let us assume, by contradiction, that there exists a sequence $\omega_{n}$ such that

$$
\int_{\omega_{n}} g^{2} d x \geqslant 1 \text { and } \int_{\partial \omega_{n}} g d \mathcal{H}^{N-1} \leqslant \frac{1}{n}
$$

For any $R$, let $B_{R}$ be the ball centered at $O$ with radius $R$ and

$$
g_{R}=\min \left\{g(x): x \in \mathbb{R}^{N},|x|=R\right\} .
$$

We fix $R>0$ such that $\int_{B_{R}} g^{2} d x<1 / 2$. We have

$$
\left|\partial \omega_{n} \backslash B_{R}\right| g_{R} \leqslant \int_{\partial \omega_{n} \backslash B_{R}} g d \mathcal{H}^{N-1} \leqslant \frac{1}{n} .
$$

Thus $\left|\partial \omega_{n} \backslash B_{R}\right| \leqslant \frac{1}{n g_{R}}$. By the Relative Isoperimetric Inequality on $\mathbb{R}^{N} \backslash B_{R}$, see for instance [14], there exists a positive constant $c_{0}$ such that

$$
\left|\omega_{n} \backslash B_{R}\right| \leqslant c_{0}\left|\partial \omega_{n} \backslash B_{R}\right|^{\frac{N}{N-1}} .
$$

This implies that $\left|\omega_{n} \backslash B_{R}\right|$ can be chosen smaller than $1 / 2 M$ where $M=\max _{D} g^{2}$, since $\left|\partial \omega_{n} \backslash B_{R}\right|$ tends to zero. Hence

$$
1 \leqslant \int_{\omega_{n}} g^{2} d x=\int_{\omega_{n} \backslash B_{R}} g^{2} d x+\int_{\omega_{n} \cap B_{R}} g^{2} d x<\frac{1}{2}+\frac{1}{2},
$$

which is a contradiction. 
REMARK 2.7 By homogeneity, statement (28) of Lemma 2.6 can also be written as:

$$
\exists C>0 \text {, such that } \forall \omega \subset D, \quad \int_{\partial \omega} g d \mathcal{H}^{N-1} \geqslant C\left(\int_{\omega} g^{2} d x\right)^{\frac{\alpha+N-1}{2 \alpha+N}} .
$$

Notice that (29) is a kind of weighted isoperimetric inequality and has its own importance: an interesting question would be to determine its optimal domains. Using the theory of quasiminimizers, one should be able to prove that such an optimal domain $\omega^{*}$ is regular. Now, the differentiation with respect to the domain would give as first order optimality condition:

$$
H g+\frac{\partial g}{\partial n}=\gamma g^{2} \text { on } \partial \omega^{*},
$$

where $H$ is the mean curvature of the boundary of $\omega^{*}$ and $\gamma$ a Lagrange multiplier.

2.1.4 Details of the proof of regularity. Let us fix a ball $B\left(x_{0}, r\right)$ with $x_{0}$ in $D$ and, as a test function in Lemma 2.5, let us choose $v$ defined as $v=u$ in $\mathbb{R}^{N} \backslash \overline{B\left(x_{0}, r\right)}$ and $v$ solution of

$$
\begin{cases}-\Delta v=1 & \text { in } B\left(x_{0}, r\right) \\ v=u & \text { on } \partial B\left(x_{0}, r\right),\end{cases}
$$

inside the ball. By the maximum principle $v>0$ in $B\left(x_{0}, r\right)$ and, therefore,

$$
\Omega_{v}=\Omega_{u} \cup B\left(x_{0}, r\right),
$$

where we recall that $\Omega_{v}=\{x: v(x)>0\}$. Thus, since $\int_{\Omega_{u}} g^{2}(x) d x=1$, we have

$$
0 \leqslant \int_{\Omega_{v}} g^{2}(x) d x-1 \leqslant \int_{B\left(x_{0}, r\right)} g^{2} \leqslant C r^{N} .
$$

It follows, from Lemma 2.5, that $u$ satisfies

$$
\int_{B\left(x_{0}, r\right)}|\nabla(u-v)|^{2} \leqslant \lambda C r^{N}
$$

and, by classical regularity results (see, e.g., Theorem 3.5.2 in [28]), $u$ is Hölder continuous on $D$. Consequently the set $\Omega_{u}$ is open as claimed at the beginning of the proof. In particular, following [9], we can prove that $u$ is Lipschitz on $D$.

Let us now study $\Delta u+\chi \Omega_{u}$. The fact that $\Delta u+1=0$ on $\Omega_{u}$ (in the sense of distributions) is easily obtained using perturbations of the kind $v=u+t \varphi$ with $\varphi \in C_{0}^{\infty}\left(\Omega_{u}\right)$. Then, following step by step [8, Theorem 2.2,Proposition 2.3], one can prove that $\Delta u+\chi \Omega_{\Omega_{u}}=\mu$, where $\mu$ is a (positive) Radon measure, supported in $\partial \Omega_{u}$ and absolutely continuous with respect to the Hausdorff measure $\mathcal{H}^{N-1}$ in $D$. Then, using a blow-up technique near the boundary points of $\partial \Omega_{u}$, we prove more precisely like in $[8$, Theorem 5.1] that

$$
\Delta u+\chi_{\Omega_{u}}=g \mathcal{H}^{N-1}\left\lfloor\partial \Omega_{u} .\right.
$$

We need for that purpose that $g$ is estimated from below: $g(x) \geqslant c>0$ which is true as soon as we are far from the origin. We can conclude using [19, Theorem 2.13, Theorem 2.17], at least outside the origin where $g=0$. 


\section{Basic properties}

In this section, we prove some basic properties of the solution to Problem (6) or (11). For that purpose we will need in the sequel to assume that the solution $\Omega$ is everywhere regular; then we set:

$$
\text { The solution } \Omega \text { of problem (6) or (11) is } C^{1, \beta} \text {. }
$$

According to Theorem 2.1, property (R) is true in dimension 2 and in higher dimension it consists in assuming that the singular part of the boundary is empty.

The first property is a somewhat technical property which will be necessary in many cases in the sequel. It states that the origin $O$ is inside the domain (at least when $\alpha>1$ ) and it is not surprising of course. Indeed, since $g$ is increasing with respect to $|x|$, one can easily imagine that translating a domain $\Omega$ toward the origin should make $\phi(\Omega)$ smaller while the torsion remains unchanged. Unfortunately we can prove such a property only for $\alpha>1$, as most of our next results.

Proposition 3.1 Let $\Omega$ be a solution of the minimization problem (11) satisfying (R) and assume that the homogeneity degree of $g$ satisfies $\alpha>1$. Then the origin $O$ belongs to $\Omega$.

Proof. Let us begin by proving that $O$ cannot be in $\mathbb{R}^{N} \backslash \bar{\Omega}$. Indeed in this case, there would exist a ball $B_{\varepsilon}=B(O, \varepsilon)$ of small radius $\varepsilon$ and center at the origin, such that $B(O, \varepsilon) \subset \mathbb{R}^{N} \backslash \bar{\Omega}$. Let $\Omega_{\varepsilon}=\Omega \cup B_{\varepsilon}$. We have immediately

$$
\phi\left(\Omega_{\varepsilon}\right)=\phi(\Omega)+\varepsilon^{2 \alpha+N} \phi\left(B_{1}\right),
$$

where $B_{1}$ is the unit ball centered at $O$. In the same way,

$$
J\left(\Omega_{\varepsilon}\right)=J(\Omega)+\varepsilon^{N+2} J\left(B_{1}\right) .
$$

Therefore, since $\alpha>1$ we get the following expansion

$$
\phi\left(\Omega_{\varepsilon}\right)^{-\frac{2+N}{2 \alpha+N}} J\left(\Omega_{\varepsilon}\right)=\phi(\Omega)^{-\frac{2+N}{2 \alpha+N}} J(\Omega)\left[1+\varepsilon^{N+2} \frac{J\left(B_{1}\right)}{J(\Omega)}+o\left(\varepsilon^{N+2}\right)\right],
$$

which contradicts the optimality of $\Omega$ since, as $J\left(B_{1}\right)<0$, it implies $J\left(\Omega_{\varepsilon}\right)<J(\Omega)$. Continuing to argue by contradiction, let us now assume that $O \in \partial \Omega$. According to assumption (R) $\partial \Omega$ satisfies an exterior cone condition at $O$ : there exists a cone $C_{\varepsilon}=\varepsilon_{\varepsilon} C_{1}$ of vertex $O$ and size $\varepsilon$ such that $C_{\varepsilon} \cap \Omega=\emptyset$. Thus we can do exactly the same construction as before considering $\Omega_{\varepsilon}=\Omega \cup C_{\varepsilon}$ and we conclude in the same way.

Next we prove the monotonicity of the solutions of (6) with respect to $g$.

THEOREM 3.2 Let $g_{1}, g_{2}$ satisfy (5) with $\alpha>1$ and let $\Omega_{1}, \Omega_{2}$ be solutions to Problem (6) related to $g_{1}$ and $g_{2}$, respectively, satisfying (R). Assume $g_{1}(x) \geqslant g_{2}(x)$ for every $x \in \mathbb{R}^{N}$, then $\Omega_{1} \subseteq \Omega_{2}$.

Proof. Assume by contradiction $\Omega_{1} \not \subset \Omega_{2}$ and consider $t \Omega_{1}$ with

$$
t=\sup \left\{s>0: s \Omega_{1} \subseteq \Omega_{2}\right\} .
$$

Then $0<t<1$ (notice that $t>0$ comes from Proposition 3.1) and there exists $\bar{x} \in \partial t \Omega_{1} \cup \partial \Omega_{2}$, with $v_{t \Omega_{1}}(\bar{x})=v_{\Omega_{2}}(\bar{x})=v$, where $v_{\Omega}(x)$ denotes the outer unit normal to $\partial \Omega$ at $x$ and $v \in S^{N-1}$. 


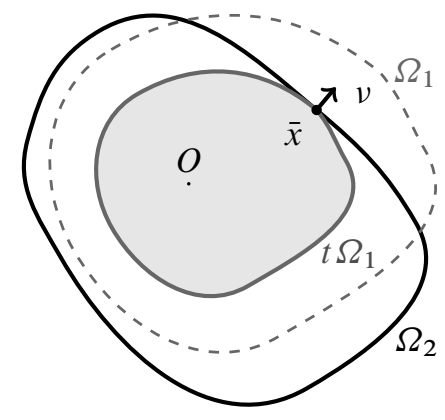

FIG. 1. $t \Omega_{1} \subseteq \Omega_{2}$ with $\bar{x} \in \partial\left(t \Omega_{1}\right) \cap \partial \Omega_{2}$

We want to compare $u_{t \Omega_{1}}$ and $u_{\Omega_{2}}$, the stress functions of $t \Omega_{1}$ and $\Omega_{2}$, respectively. Notice that $u_{t \Omega_{1}}(x)=t^{2} u_{\Omega_{1}}\left(\frac{x}{t}\right)$. Define $w=u_{\Omega_{2}}-u_{t \Omega_{1}}$; it satisfies

$$
\begin{cases}\Delta w=0 & \text { in } t \Omega_{1}, \\ w \geqslant 0 & \text { on } \partial t \Omega_{1}, \\ w(\bar{x})=0 . & \end{cases}
$$

Hence by Hopf Lemma, it holds $\frac{\partial w}{\partial v}(\bar{x})>0$. Notice that

$$
\frac{\partial w}{\partial v}(\bar{x})=\left|\nabla u_{\Omega_{2}}(\bar{x})\right|-\left|\nabla u_{t \Omega_{1}}(\bar{x})\right|=g_{2}(\bar{x})-\operatorname{tg}_{1}\left(\frac{\bar{x}}{t}\right),
$$

since $v$ is parallel to $\nabla u_{\Omega_{2}}(\bar{x}), \nabla u_{t} \Omega_{1}(\bar{x})$ and $\left|\nabla u_{t \Omega_{1}}(\bar{x})\right|=t\left|\nabla u_{\Omega_{1}}\left(\frac{\bar{x}}{t}\right)\right|$, with $\frac{\bar{x}}{t} \in \partial \Omega_{1}$. Hence, by the homogeneity of $g_{1}$, and the fact that $t<1, \alpha>1$, we get

$$
g_{2}(\bar{x})>\operatorname{tg}\left(\frac{\bar{x}}{t}\right)=t^{1-\alpha} g_{1}(\bar{x})>g_{1}(\bar{x}),
$$

which contradicts the assumption $g_{1} \geqslant g_{2}$.

REMARK 3.3 We can prove the above theorem under some weaker assumptions, precisely it is sufficient that at least one between $g_{1}$ and $g_{2}$ satisfies (5), with $\alpha>1$. In such a case we have however to assume $O$ belongs to the interior of both $\Omega_{1}$ and $\Omega_{2}$ and that they are bounded. In case $g_{1}$ is the function satisfying assumption (5), the proof is precisely the same as above. Notice that in this case $O \in \Omega_{1}$ and $\Omega_{1}$ bounded are pleonastic assumptions since they implied by Proposition 3.1 and he result os Section 2, respectively. If $g_{2}$ satisfies (5) with $\alpha>1$ instead of $g_{1}$, then we argue again by contradiction, very similarly as before (in this case $O \in \Omega_{2}$ and $\Omega_{2}$ bounded are pleonastic). Indeed if $\Omega_{1} \not \subset \Omega_{2}$, consider $t \Omega_{2}$ with

$$
t=\inf \left\{s>0: \Omega_{1} \subseteq s \Omega_{2}\right\}
$$

Then $t>1$ and there exists $\bar{x} \in \partial \Omega_{1} \cup \partial\left(t \Omega_{2}\right)$, with $v_{\Omega_{1}}(\bar{x})\left\|v_{t} \Omega_{2}(\bar{x})\right\| v$, where $v_{\Omega}(x)$ denotes the outer unit normal to $\partial \Omega$ at $x$ and $v \in S^{N-1}$. Then we can proceed in the same way as before. 
As a natural straightforward corollary of the previous theorem, the uniqueness of the solution follows.

THEOREM 3.4 If $g$ satisfies assumptions (5) for $\alpha>1$, then there is a unique solution satisfying (R).

\section{Starshapedness and connectedness}

We recall that a set $\Omega$ is said starshaped with respect to a point $x_{0} \in \Omega$ if

$$
t\left(x-x_{0}\right)+x_{0} \in \Omega \quad \text { for every } x \in \Omega \text { and every } t \in[0,1] .
$$

When $x_{0}=O$ we simply say that $\Omega$ is starshaped.

THEOREM 4.1 If $\Omega$ satisfy (R) and $g$ satisfies assumptions (5) for $\alpha>1$, then $\Omega$ is starshaped (with respect to $O$ ).

Proof. The proof is similar to that one of Theorem 3.2.

By contradiction, assume $\Omega$ is not starshaped and let

$$
t=\inf \{s \in[0,1]: s \Omega \not \subset \Omega\} .
$$

There exists $\bar{x} \in \partial(t \Omega) \cap \partial \Omega$ (see Figure 2). Notice that $0<t<1$ since the origin belongs to $\Omega$, and $\Omega$ is bounded and not starshaped. In a similar way as before, consider the function

$$
u_{t}(x)=t^{2} u_{\Omega}\left(\frac{x}{t}\right) .
$$

Then $u_{t}$ is the stress function of $t \Omega$ and whence

$$
\begin{cases}-\Delta w=0 & \text { in } t \Omega \\ w \geqslant 0 & \text { on } \partial(t \Omega) \\ w(\bar{x})=0, & \end{cases}
$$

where $w=u_{\Omega}-u_{t}$.

Then

$$
\frac{\partial w}{\partial v}(\bar{x}) \leqslant 0,
$$

where $v$ is the outer unit normal at $\bar{x}$ of $\Omega$ and $t \Omega$. On the other hand

$$
\frac{\partial w}{\partial v}(\bar{x})=\left|\nabla u_{t}(\bar{x})\right|-\left|\nabla u_{\Omega}(\bar{x})\right|,
$$

then (30) reads as

$$
\left|\nabla u_{t}(\bar{x})\right| \leqslant|\nabla u(\bar{x})|=g(\bar{x}) .
$$

Moreover a straightforward calculation gives

$$
\nabla u_{t}(x)=t \nabla u_{\Omega}\left(\frac{x}{t}\right) \quad \text { for } x \in t \Omega,
$$

which entails

$$
\left|\nabla u_{t}(\bar{x})\right|=t\left|\nabla u_{\Omega}(\bar{x} / t)\right|=\operatorname{tg}(\bar{x} / t)=t^{1-\alpha} g(\bar{x})>g(\bar{x}),
$$

for $1-\alpha<0, t<1$ and $\bar{x} \neq 0$ (then $g(\bar{x})>0$ ).

The latter contradicts (31) and the proof of starshapedness is concluded. 


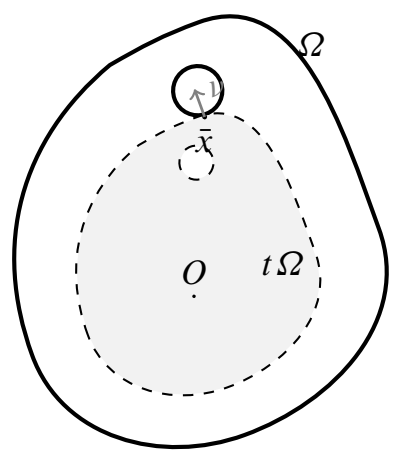

FIG. 2. $t=\inf \{s \in[0,1]: s \Omega \nsubseteq \Omega\}$.

Starshapedness obviously implies connectedness. Then when $\alpha>1$ the solution is connected. We are able to prove this property also when $\alpha<1$.

Proposition 4.2 Let $\alpha \neq 1$ and $\Omega$ be a solution of the minimization problem (11) or (12). Then $\Omega$ is connected.

Proof. For $\alpha>1$ the thesis follows from Theorem 4.1 as already pointed out. It is then sufficient to consider the case $\alpha<1$. Let us assume, by contradiction, that the solution $\Omega$ is not connected and let us write it as

$$
\Omega=\Omega_{1} \cup \Omega_{2} \text {, with } \Omega_{1} \cap \Omega_{2}=\emptyset,\left|\Omega_{1}\right|>0,\left|\Omega_{2}\right|>0 .
$$

Since $g$ is positive outside 0 , we have $\phi\left(\Omega_{i}\right)>0$ for $i=1,2$. Let us denote by $M$ the value of the minimum

$$
M=\phi(\Omega)^{-\frac{2+N}{2 \alpha+N}} J(\Omega)<0 .
$$

For each component, we have $\phi\left(\Omega_{i}\right)^{-\frac{2+N}{2 \alpha+N}} J\left(\Omega_{i}\right) \geqslant M, i=1$, 2. Now, $J(\Omega)=J\left(\Omega_{1}\right)+J\left(\Omega_{2}\right)$ and $\phi(\Omega)=\phi\left(\Omega_{1}\right)+\phi\left(\Omega_{2}\right)$. Therefore

$$
J(\Omega) \geqslant M\left(\phi\left(\Omega_{1}\right)^{\frac{2+N}{2 \alpha+N}}+\phi\left(\Omega_{2}\right)^{\frac{2+N}{2 \alpha+N}}\right)>M\left(\phi\left(\Omega_{1}\right)+\phi\left(\Omega_{2}\right)\right)^{\frac{N+2}{2 \alpha+N}}=M \phi(\Omega)^{\frac{N+2}{2 \alpha+N}},
$$

the strict inequality coming from the fact that $\alpha<1$ (whence $\frac{N+2}{2 \alpha+N}>1$ ), $\phi\left(\Omega_{i}\right)>0$ for $i=1,2$ and $M<0$. This clearly leads to a contradiction with (32).

\section{Convexity}

Let us recall some definitions which will be useful later on. A lower semicontinuous function $u$ : $\mathbb{R}^{N} \rightarrow \mathbb{R} \cup\{ \pm \infty\}$ is said quasi-convex if it has convex sublevel sets, or, equivalently, if

$$
u\left((1-\lambda) x_{0}+\lambda x_{1}\right) \leqslant \max \left\{u\left(x_{0}\right), u\left(x_{1}\right)\right\},
$$

for every $\lambda \in[0,1]$, and every $x_{0}, x_{1} \in \mathbb{R}^{N}$. If $u$ is defined only in a proper subset $\Omega$ of $\mathbb{R}^{n}$,we extend $u$ as $+\infty$ in $\mathbb{R}^{n} \backslash \Omega$ and we say that $u$ is quasi-convex in $\Omega$ if such an extension is quasiconvex in $\mathbb{R}^{N}$. In an analogous way, $u$ is quasi-concave if $-u$ is quasi-convex, i.e., if it has convex 
superlevel sets. Obviously, if $u$ (or any one of its powers) is convex (concave) then it is quasi-convex (quasi-concave) but the reverse is not necessarily true.

REMARK 5.1 Notice that, due to the $\alpha$-homogeneity, the quasi-convexity of $g$ is equivalent (for $\alpha>0$ ) to the following apparently stronger property:

$$
g^{1 / \alpha} \text { is convex. }
$$

Indeed, notice that $h(x)=g^{\frac{1}{\alpha}}(x)$ is homogeneous of degree one and it is quasi-convex. Fix $x_{0}, x_{1} \in \mathbb{R}^{N}$ and consider $\lambda \in[0,1]$. We want to prove that

$$
\frac{h\left((1-\lambda) x_{0}+\lambda x_{1}\right)}{(1-\lambda) h\left(x_{0}\right)+\lambda h\left(x_{1}\right)} \leqslant 1 .
$$

Denote by

$$
\xi=\frac{\lambda h\left(x_{1}\right)}{(1-\lambda) h\left(x_{0}\right)+\lambda h\left(x_{1}\right)}
$$

using the quasi-convexity and the homogeneity of $h$, we get

$$
\frac{h\left((1-\lambda) x_{0}+\lambda x_{1}\right)}{(1-\lambda) h\left(x_{0}\right)+\lambda h\left(x_{1}\right)}=h\left((1-\xi) \frac{x_{0}}{h\left(x_{0}\right)}+\xi \frac{x_{1}}{h\left(x_{1}\right)}\right) \leqslant \max \left\{h\left(\frac{x_{0}}{h\left(x_{0}\right)}\right), h\left(\frac{x_{1}}{h\left(x_{1}\right)}\right)\right\}=1 .
$$

THEOREM 5.2 Let $g$ be a quasi-convex and homogeneous function of degree $\alpha \geqslant 2$, with $g(x)>0$ for $x \neq 0$. Assume that $\Omega$ satisfies (R), then $\Omega$ is convex.

Proof. The proof follows the same lines of that of starshapedness. By contradiction, assume $\Omega$ is not convex and let $\Omega^{*}$ be its convex hull. Denote by $u_{\Omega}$ the solution of (2) and by $u_{\Omega^{*}}$ the stress function of $\Omega^{*}$. Let

$$
t=\sup \left\{s \in[0,1]: s \Omega^{*} \subset \Omega\right\}
$$

(see Figure 3) and let

$$
\bar{x} \in \partial\left(t \Omega^{*}\right) \cap \partial \Omega .
$$

Notice that $t>0$ since $0 \in \Omega$ and $\Omega^{*}$ is bounded (for $\Omega$ is bounded) while $t<1$ for $\Omega$ is not convex. It is easily seen that

$$
u_{t}(x)=t^{2} u_{\Omega^{*}}\left(\frac{x}{t}\right),
$$

solves (2) with $t \Omega^{*}$ in place of $\Omega$, (that is $u_{t}$ is the stress function of $t \Omega^{*}$ ) whence

$$
\begin{cases}-\Delta w=0 & \text { in } t \Omega^{*}, \\ w \geqslant 0 & \text { on } \partial\left(t \Omega^{*}\right), \\ w(\bar{x})=0, & \end{cases}
$$

where $w=u_{\Omega}-u_{t}$.

Then

$$
\frac{\partial w}{\partial v}(\bar{x}) \leqslant 0,
$$




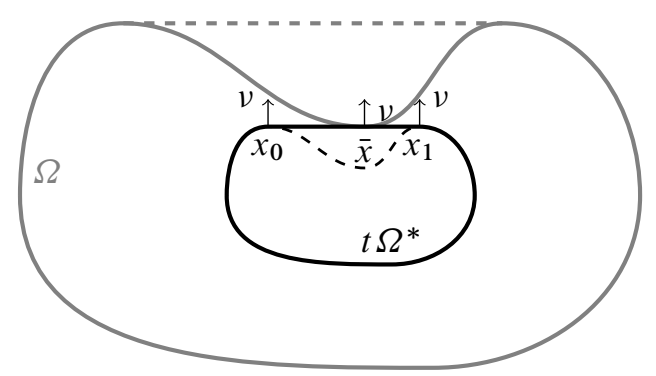

FIG. 3. $t=\sup \left\{s \in[0,1]: s \Omega^{*} \subseteq \Omega\right\}$

where $v$ is the common outer unit normal at $\bar{x}$ of $\Omega$ and $t \Omega^{*}$. On the other hand

$$
\frac{\partial w}{\partial v}(\bar{x})=\left|\nabla u_{t}(\bar{x})\right|-\left|\nabla u_{\Omega}(\bar{x})\right|,
$$

then (33) reads

$$
\left|\nabla u_{t}(\bar{x})\right| \leqslant\left|\nabla_{\Omega} u(\bar{x})\right|
$$

We will contradict the latter by giving estimates of the gradient of $u_{t}$ which show that the reverse must be true. To do this we need the following property, whose proof is given at the end of the section.

LEMma 5.3 If $\bar{x} \in \partial \Omega^{*} \backslash \partial \Omega, m \geqslant 2$ and $x_{1}, \ldots, x_{m} \in \partial \Omega \cap \partial \Omega^{*}$ are such that $\bar{x}=\sum_{i=1}^{m} \lambda_{i} x_{i}$, for some $\lambda_{1}, \ldots, \lambda_{m} \in[0,1]$ with $\sum_{i=1}^{m} \lambda_{i}=1$, then

$$
\left|\nabla u_{\Omega^{*}}(\bar{x})\right| \geqslant\left(\sum_{i=1}^{N} \lambda_{i} \sqrt{\left|\nabla u_{\Omega}\left(x_{i}\right)\right|}\right)^{2} .
$$

With Lemma 5.3 at hands, it is easy to prove that (34) can not hold true. Indeed a straightforward calculation gives

$$
\nabla u_{t}(x)=t \nabla u_{\Omega^{*}}\left(\frac{x}{t}\right) \text { for } x \in t \Omega^{*} .
$$

Notice that, as $\bar{x} \in \partial\left(t \Omega^{*}\right)$, there exist $x_{1}, \ldots, x_{N} \in \partial(t \Omega)$ (some of which possibly coinciding) and $\lambda_{1}, \ldots, \lambda_{N} \in[0,1]$ such that $\sum_{i=1}^{N} \lambda_{i}=1$ and $\bar{x}=\sum_{i=1}^{N} \lambda_{i} x_{i}$ and $v(\bar{x})=v\left(x_{1}\right)=\cdots=$ $v\left(x_{N}\right)$, where $v(x)$ indicates the outer unit normal vector to $t \Omega^{*}$ or $t \Omega$ at the involved point $x$ (see Proposition 3.1 of [17] for instance). Moreover, since $\bar{x} \in \partial\left(t \Omega^{*}\right) \backslash \partial(t \Omega)$, we have by Lemma 5.3

$$
\begin{aligned}
\left|\nabla u_{t}(\bar{x})\right|=t\left|\nabla u_{\Omega^{*}}(\bar{x} / t)\right| & \geqslant t\left(\sum_{i=1}^{N} \lambda_{i} \sqrt{\left|\nabla u_{\Omega}\left(x_{i} / t\right)\right|}\right)^{2} \\
& =t\left(\lambda_{i} \sqrt{g\left(\frac{x_{i}}{t}\right)}\right)^{2} \\
& =t^{1-\alpha}\left(\sum_{i=1}^{N} \lambda_{i} \sqrt{g\left(x_{i}\right)}\right)^{2}
\end{aligned}
$$


On the other hand, Remark 5.1 yields

$$
g(\bar{x}) \leqslant\left(\sum_{i=1}^{N} \lambda_{i} g\left(x_{i}\right)^{\frac{1}{\alpha}}\right)^{\alpha},
$$

and then

$$
g(\bar{x}) \leqslant\left(\sum_{i=1}^{N} \lambda_{i} g\left(x_{i}\right)^{\frac{1}{2}}\right)^{2}
$$

since $\alpha \geqslant 2$. We finally get

$$
\left|\nabla u_{t}(\bar{x})\right| \geqslant t^{1-\alpha} g(\bar{x})>g(\bar{x})=|\nabla u(\bar{x})|,
$$

where the strict inequality holds because $t<1, \alpha>1$ and $g(\bar{x})>0$, since $\bar{x} \neq 0$ for $0 \in \Omega$. The latter contradicts (34) and the proof is concluded.

Now we give the proof of Lemma 5.3.

Proof of Lemma 5.3. We use the same notations and a similar construction as in the proof of Theorem 5.2. So, let $\Omega^{*}$ be the convex hull of $\Omega$ and let $u_{\Omega^{*},} u_{\Omega}$ be their stress functions, respectively. By Comparison Principle we have

$$
u_{\Omega} \leqslant u_{\Omega^{*}} \text { in } \Omega .
$$

Since

$$
u_{\Omega}=u_{\Omega^{*}} \quad \text { on } \partial \Omega^{*} \cap \partial \Omega,
$$

equation (37) entails

$$
\left|\nabla u_{\Omega^{*}}(x)\right| \geqslant\left|\nabla u_{\Omega}(x)\right| \text { for every } x \in \partial \Omega^{*} \cap \partial \Omega .
$$

In particular we have

$$
\left|\nabla u_{\Omega^{*}}\left(x_{i}\right)\right| \geqslant\left|\nabla u_{\Omega}\left(x_{i}\right)\right| \text { for } i=1, \ldots, m \text {. }
$$

Next we prove that

$$
\left|\nabla u_{\Omega^{*}}(\bar{x})\right| \geqslant\left(\sum_{i=1}^{m} \sqrt{\left|\nabla u_{\Omega^{*}}\left(x_{i}\right)\right|}\right)^{2} .
$$

Notice that $\partial \Omega^{*}$ inherits $C^{1}$ regularity from $\partial \Omega$ and the outer normal directions $v\left(x_{1}\right), \ldots, v\left(x_{m}\right)$ to $\partial \Omega^{*}$ at the points $x_{1}, \ldots, x_{m}$ are all coinciding with the outer normal direction $v$ to $\partial \Omega^{*}$ at the point $\bar{x}$ and then $\nabla u_{\Omega^{*}}(\bar{x}), \nabla u_{\Omega^{*}}\left(x_{1}\right), \ldots, \nabla u_{\Omega^{*}}\left(x_{m}\right)$ are all parallel to $-v$. By the convexity of $\Omega^{*}$ it is well known that $u_{\Omega^{*}}$ is $\frac{1}{2}$-concave (see $[13,26,27]$ ), that is

$$
v=\sqrt{u \Omega^{*}} \text { is concave in } \bar{\Omega}^{*} .
$$

Moreover, since

$$
\sum_{i=1}^{m} \lambda_{i}\left(x_{i}-t v\right)=\bar{x}-t v
$$


for every small enough $t>0$ we have

$$
u_{\Omega^{*}}(\bar{x}-t v)=v(\bar{x}-t v)^{2} \geqslant\left(\sum_{i=1}^{m} \lambda_{i} v\left(x_{i}-t v\right)\right)^{2} .
$$

By the definition of $v$ the latter reads as

$$
\frac{u_{\Omega^{*}}(\bar{x}-t v)}{t} \geqslant\left(\sum_{i=1}^{m} \lambda_{i} \sqrt{\frac{u_{\Omega *}\left(x_{i}-t v\right)}{t}}\right)^{2} .
$$

Passing to the limit as $t \rightarrow 0^{+}$and taking into account that

$$
u_{\Omega^{*}}(\bar{x})=u_{\Omega^{*}}\left(x_{i}\right)=0, \quad i=1, \ldots, m,
$$

we get (39).

Coupling (39) and (38) gives (35) and the proof is concluded.

\section{Symmetries}

In this section we prove that some symmetry properties of $g$ are inherited by the solution $\Omega$. In particular in Theorem 6.1 we consider the radial case, while Theorem 6.6 treats the Steiner symmetric case. In both cases the presented technique is based on rearrangements of sets and functions.

THEOREM 6.1 If $g$ is radial and satisfies assumptions (5), then the solution $\Omega$ to the minimization problem (11) is a ball.

Notice that a radial solution can always be explicitly computed. The above result states that in fact not radial solutions cannot exist. Let us remark that for $\alpha>1$ this can also be seen as a straightforward corollary of Theorem 3.4.

Proof. Let us denote by $\Omega^{\#}$ the ball of the same volume as $\Omega$ and center at $O$. Assume $\Omega \backslash \Omega^{\#}$ to be a set of positive measure. We are going to reach a contradiction proving that the value $J\left(\Omega^{\#}\right)$ is strictly better than the minimum value $J(\Omega)$.

Notice that, since $g$ is radial and increasing in each direction, we have

$$
\Omega^{\#}=\left\{x \in \mathbb{R}^{N}: g(x) \leqslant \bar{t}\right\},
$$

for some $\bar{t}>0$.

Hence

$$
\inf _{x \in \Omega \backslash \Omega^{\#}} g^{2}(x)>\bar{t}=\sup _{x \in \Omega^{\#} \backslash \Omega} g^{2}(x),
$$




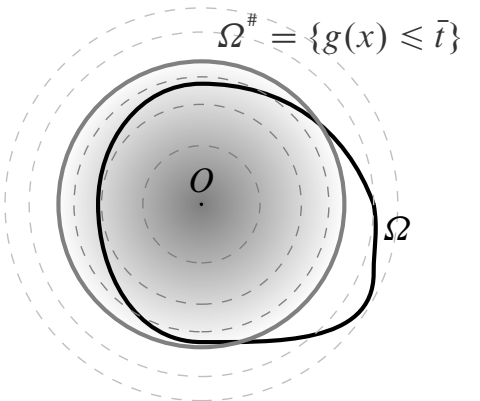

FIG. 4. The level lines of $g$ are monotone increasing concentric balls

which implies $\phi\left(\Omega^{\#}\right)<\phi(\Omega)$, since

$$
\begin{aligned}
\phi\left(\Omega^{\#}\right) & \leqslant \int_{\Omega^{\#} \cap \Omega} g^{2}(x) d x+\left|\Omega^{\#} \backslash \Omega\right| \sup _{x \in \Omega^{\#} \backslash \Omega} g^{2}(x) \\
& <\int_{\Omega^{\#} \cap \Omega} g^{2}(x) d x+\left|\Omega^{\#} \backslash \Omega\right| \inf _{x \in \Omega \backslash \Omega^{\#}} g^{2}(x) \\
& \leqslant \int_{\Omega^{\#} \cap \Omega} g^{2}(x) d x+\int_{\Omega \backslash \Omega^{\#}} g^{2}(x) d x \\
& =\phi(\Omega) .
\end{aligned}
$$

In order to compare the stress function of $\Omega$ with that of $\Omega^{\#}$, we compare both of them with a rearrangement of $u_{\Omega}$. More precisely, let $u^{\#}$ be the Schwarz symmetric of the stress function $u_{\Omega}$, that is the radial function whose sublevel sets are concentric balls of the same measure than the corresponding sublevel sets of $u$ (that is $\left|\left\{u^{\#}<t\right\}\right|=\left|\left\{u_{\Omega}<t\right\}\right|$ ). We compare $u^{\#}$ with the stress functions of $\Omega^{\#}, u_{\Omega^{\#}}$ (notice that it is a radial function too) and that of $\Omega$. Recalling the characterization of stress functions in (4), we have

$$
J\left(\Omega^{\#}\right) \leqslant \frac{1}{2} \int_{\Omega^{\#}}\left\|D u^{\#}\right\|^{2} d x-\int_{\Omega^{\#}} u^{\# 2} d x ;
$$

moreover, by classical results (see for instance [25]), it holds

$$
\begin{gathered}
\int_{\Omega^{\#}} u^{\# 2} d x=\int_{\Omega} u_{\Omega}^{2} d x, \\
\int_{\Omega^{\#}}\left\|D u^{\#}\right\|^{2} d x<\int_{\Omega}\left\|D u_{\Omega}\right\|^{2} d x,
\end{gathered}
$$

where the strict inequality holds since $u_{\Omega}$ is not radial (otherwise $\Omega$ would be a ball) and hence it does not coincides with $u^{\#}$ (see [25] Corollary 2.33). These entail $J\left(\Omega^{\#}\right)<J(\Omega)$, which is a contradiction. This shows that $\Omega$ is a ball, up to a zero measure set.

Notice that, under stronger assumptions a similar result on the symmetry of the solution to the torsion problem has been proved by A. Greco in [20]. More precisely he considered Problem (6) 
with $g(x)=c|x|$, and he proved that if a solution exists and the set $\Omega$ contains the origin, then the set must be a ball.

We want to use Theorem 7.1 to estimate the stability of the radial setting. Roughly speaking we deal with the following questions: If, in some sense, $g$ is close to be a radial function, is $\Omega$ close (in a suitable sense) to be a ball? And how does the distance of $\Omega$ from the ball shape depend on the distance of $g$ from the radial shape?

It is then necessary to specify which kind of distance is convenient to use to measure the closeness of $g$ to be radial.

We present two stability results which are in fact corollaries of Theorem 3.2. More precisely, we consider two different kinds of distances of functions: in Proposition 6.2 we ask $g$ to be quasi radial in the $L^{\infty}$ norm, while in Proposition 6.3 the distance between the function $g$ and a radial function is controlled in terms of the $\alpha$-homogeneous sublinear function $|x|^{\alpha}$. Notice that this latter is in fact quite natural since the space of $\alpha$-homogeneous functions is considered.

Proposition 6.2 Let $g, h$ satisfy (5) with $\alpha>1$ and assume $h$ to be radial with

$$
|g(x)-h(x)| \leqslant \varepsilon,
$$

for every $x \in \mathbb{R}^{N}$ and some $\varepsilon>0$. Let $\Omega$ be the solution of (6) related to $g$, then there exist $R_{\varepsilon}>r_{\varepsilon}>0$ such that $B\left(O, r_{\varepsilon}\right) \subseteq \Omega \subseteq B\left(O, R_{\varepsilon}\right)$ with $\left|R_{\varepsilon}-r_{\varepsilon}\right|=\mathscr{O}(\varepsilon)$.

Proof. Since the function $h$ is $\alpha$-homogeneous and radial, there exists a positive constant $k$ such that $h(x)=k|x|^{\alpha}$, where in fact $k=\left.h\right|_{S^{N-1}}$. Define

$$
h_{-}(x)=\frac{h(x)}{1-\varepsilon}, \quad h_{+}(x)=\frac{h(x)}{1+\varepsilon} ;
$$

it holds $h_{+} \leqslant g \leqslant h_{-}$since

$$
\left\{x \in \mathbb{R}^{N}: h_{+}(x) \leqslant 1\right\} \subseteq\left\{x \in \mathbb{R}^{N}: g(x) \leqslant 1\right\} \subseteq\left\{x \in \mathbb{R}^{N}: h_{-}(x) \leqslant 1\right\} .
$$

Moreover $h_{-}, h_{+}$satisfy the hypothesis of Theorem 3.2 and hence

$$
\Omega_{-} \subseteq \Omega \subseteq \Omega_{+},
$$

where $\Omega_{-}, \Omega_{+}$are the solutions to (6) related to $h_{-}, h_{+}$, respectively.

By Theorem 6.1 there exist $R_{\varepsilon}>r_{\varepsilon}>0$ such that $\Omega_{-}=B\left(O, r_{\varepsilon}\right)$ and $\Omega_{+}=B\left(O, R_{\varepsilon}\right)$. In particular, solving explicitly Problem 6 in the radial homogeneous case, we get

$$
R_{\varepsilon}=\left(\frac{1+\varepsilon}{k N}\right)^{\frac{1}{\alpha-1}}, \quad r_{\varepsilon}=\left(\frac{1-\varepsilon}{k N}\right)^{\frac{1}{\alpha-1}},
$$

since the stress function of a ball $B(O, \rho)$ is $u(x)=\frac{\rho^{2}-|x|^{2}}{2 N}$, with $\mid D u \|_{\partial B(O, \rho)}=\frac{\rho}{N}$. Comparing $R_{\varepsilon}$ and $r_{\varepsilon}$ we have

$$
\left|R_{\varepsilon}-r_{\varepsilon}\right|=\frac{2}{(\alpha-1)(N k)^{\frac{1}{\alpha-1}}}(\varepsilon+o(\varepsilon)),
$$

which entails the thesis. 
Proposition 6.3 Let $g, h$ satisfy (5) with $\alpha>1$ and assume $h$ to be radial with

$$
|g(x)-h(x)| \leqslant \varepsilon|x|^{\alpha},
$$

for every $x \in \mathbb{R}^{N}$ and some $\varepsilon>0$. Let $\Omega$ be the solution of (6) related to $g$. There exist $R_{\varepsilon}^{\prime}>r_{\varepsilon}^{\prime}>0$ such that $B\left(O, r_{\varepsilon}^{\prime}\right) \subseteq \Omega \subseteq B\left(O, R_{\varepsilon}^{\prime}\right)$ with $\left|R_{\varepsilon}^{\prime}-r_{\varepsilon}^{\prime}\right|=\mathscr{O}(\varepsilon)$.

Proof. As before we notice that the assumptions on $h$ implies that there exists a positive constant $k$ such that $h(x)=k|x|^{\alpha}$.

Since the stress function of a ball $B(O, \rho)$ is of the form $u(x)=\frac{1}{2 N}\left(\rho-|x|^{2}\right)$, the solutions to (6) related to $h+\varepsilon|x|^{\alpha}, h-\varepsilon|x|^{\alpha}$ are

$$
u_{+}(x)=\frac{r_{\varepsilon}^{\prime 2}-|x|^{2}}{2 N}, \quad u_{-}(x)=\frac{R_{\varepsilon}^{\prime 2}-|x|^{2}}{2 N},
$$

with $r_{\varepsilon}^{\prime}=(N(k+\varepsilon))^{-\frac{1}{\alpha-1}}, R_{\varepsilon}^{\prime}=(N(k-\varepsilon))^{-\frac{1}{\alpha-1}}$, respectively.

Then, for every $\alpha>1$, it holds $\lim _{\varepsilon \rightarrow 0^{+}} r_{\varepsilon}^{\prime}=\lim _{\varepsilon \rightarrow 0^{+}} R_{\varepsilon}^{\prime}=(k N)^{-\frac{1}{\alpha-1}}$, which entails the thesis. Notice that the value $r=(k N)^{-\frac{1}{\alpha-1}}$, corresponds to the value of the radius of the ball solution to Problem (6) related to the function $h(x)$.

REMARK 6.4 Notice that, by the homogeneity of $g$ and $h$, the condition $|g(x)-h(x)| \leqslant \varepsilon$ in Proposition 6.2 guarantees the validity of the condition $|g(x)-h(x)| \leqslant \varepsilon|x|^{\alpha}$ in Proposition 6.3. Indeed

$$
h(x)+\varepsilon|x|^{\alpha}=|x|^{\alpha}\left(h\left(\frac{x}{|x|}\right)+\varepsilon\right) \geqslant|x|^{\alpha}\left(g\left(\frac{x}{|x|}\right)\right)=g(x),
$$

and, analogously, $h(x)-\varepsilon|x|^{\alpha} \leqslant g(x)$.

However Proposition 6.2 is stronger than Proposition 6.3, depending on the value of $k>0$. In particular if $k<1-\varepsilon$, then the balls $B\left(O, r_{\varepsilon}\right), B\left(O, R_{\varepsilon}\right)$ give a better approximation to the set $\Omega$, while for $k>1+\varepsilon$, it is more convenient to compare $\Omega$ with $B\left(O, r_{\varepsilon}^{\prime}\right), B\left(O, R_{\varepsilon}^{\prime}\right)$. For $1-\varepsilon<k<1+\varepsilon$ we have $R_{\varepsilon}<R_{\varepsilon}^{\prime}$ and $r_{\varepsilon}>r_{\varepsilon}^{\prime}$.

REMARK 6.5 Since $g$ is homogeneous, it is completely described by its degree of homogeneity $\alpha$ and one of its level set, say

$$
G_{1}=\left\{x \in \mathbb{R}^{N}: g(x)<1\right\} .
$$

Indeed the sublevel set $G_{t}$ can be written in terms of $G_{1}$, see (42).

Hence the distance of $g$ to be radial can be conveniently expressed in terms of the distance of $G_{1}$ from a ball.

Propositions 6.2 and 6.3 can in fact be rewritten in terms of sublevel sets. More precisely the condition $|g-h| \leqslant \varepsilon$ becomes

$$
B\left(O,\left(\frac{1-\varepsilon}{k}\right)^{\frac{1}{\alpha}}\right) \subset G_{1} \subset B\left(O,\left(\frac{1+\varepsilon}{k}\right)^{\frac{1}{\alpha}}\right),
$$

where $k=\left.h\right|_{S^{N-1}}$, while $|g-h| \leqslant \varepsilon|x|^{\alpha}$ entails

$$
B\left(O, \frac{1}{(k+\varepsilon)^{\frac{1}{\alpha}}}\right) \subset G_{1} \subset B\left(O, \frac{1}{(k-\varepsilon)^{\frac{1}{\alpha}}}\right) .
$$


We now present another symmetry result concerning the Steiner symmetric case. More precisely, let us assume $g$ to be Steiner symmetric in the following sense:

$$
\left\{\begin{array}{l}
g \text { is symmetric with respect to }\left\{x_{N}=0\right\}, \text { and } \\
\text { the sublevel sets of } g \text { are convex in the } x_{N} \text { direction. }
\end{array}\right.
$$

Notice that this can be rephrased as

$$
g\left(x^{\prime}, x_{N}\right) \geqslant g\left(x^{\prime}, y_{N}\right), \quad \text { whenever }\left|x_{N}\right| \geqslant\left|y_{N}\right| .
$$

Theorem 6.6 Consider a function $g$ satisfying assumptions (5) and assume $g$ to be Steiner symmetric in the sense of (41). Then the solution $\Omega$ to Problem (11) is symmetric with respect to the hyperplane $\left\{x_{N}=0\right\}$.

Proof. The proof makes use of Steiner symmetrization. Let us recall some notations; for more details, we refer to [30].

We denote by $\Omega^{\prime}$ the projection of $\Omega$ onto $\mathbb{R}^{N-1}$ :

$$
\Omega^{\prime}=\left\{x^{\prime} \in \mathbb{R}^{N-1} \text { such that there exists } x_{N} \text { with }\left(x^{\prime}, x_{N}\right) \in \Omega\right\} .
$$

For $x^{\prime} \in \mathbb{R}^{N-1}$, we denote by $\Omega\left(x^{\prime}\right)$ the intersection of $\Omega$ with the line $\left\{x^{\prime}\right\} \times \mathbb{R}$; that is

$$
\Omega\left(x^{\prime}\right):=\left\{x_{N} \in \mathbb{R} \text { such that }\left(x^{\prime}, x_{N}\right) \in \Omega\right\} .
$$

Obviously $\Omega\left(x^{\prime}\right)$ is the empty set for every $x^{\prime} \in \mathbb{R}^{N-1} \backslash \Omega^{\prime}$ while

$$
\bigcup_{x^{\prime} \in \Omega^{\prime}}\left(x^{\prime} \times \Omega\left(x^{\prime}\right)\right)=\Omega .
$$

We introduce the one-dimensional set

$$
\Omega^{\star}\left(x^{\prime}\right):=\left(-\frac{1}{2}\left|\Omega\left(x^{\prime}\right)\right|, \frac{1}{2}\left|\Omega\left(x^{\prime}\right)\right|\right) ;
$$

which is a symmetric interval with the same measure as $\Omega\left(x^{\prime}\right)$. by

The Steiner symmetrized of $\Omega$ with respect to the hyperplane $\left\{x_{N}=0\right\}$ is the set $\Omega^{\star}$ defined

$$
\Omega^{\star}:=\left\{x=\left(x^{\prime}, x_{N}\right) \text { such that }-\frac{1}{2}\left|\Omega\left(x^{\prime}\right)\right|<x_{N}<\frac{1}{2}\left|\Omega\left(x^{\prime}\right)\right|, x^{\prime} \in \Omega^{\prime}\right\} .
$$

Now, since $g$ is increasing in the $x_{N}$ direction from $x_{N}=0$ and symmetric, we have for any $x^{\prime} \in \Omega^{\prime}$

$$
\begin{aligned}
\int_{\Omega\left(x^{\prime}\right)} g^{2}\left(x^{\prime}, x_{N}\right) d x_{N} & =\int_{\Omega\left(x^{\prime}\right) \cap \Omega^{\star}\left(x^{\prime}\right)} g^{2}\left(x^{\prime}, x_{N}\right) d x_{N}+\int_{\Omega\left(x^{\prime}\right) \backslash \Omega^{\star}\left(x^{\prime}\right)} g^{2}\left(x^{\prime}, x_{N}\right) d x_{N} \\
& \geqslant \int_{\Omega^{\star}\left(x^{\prime}\right) \cap \Omega\left(x^{\prime}\right)} g^{2}\left(x^{\prime}, x_{N}\right) d x_{N}+\int_{\Omega^{\star}\left(x^{\prime}\right) \backslash \Omega\left(x^{\prime}\right)} g^{2}\left(x^{\prime}, x_{N}\right) d x_{N} \\
& =\int_{\Omega^{\star}\left(x^{\prime}\right)} g^{2}\left(x^{\prime}, x_{N}\right) d x_{N} .
\end{aligned}
$$


Therefore, integrating over $\Omega^{\prime}$, we get

$$
\int_{\Omega} g^{2} d x=\int_{\Omega^{\prime}} d x^{\prime} \int_{\Omega\left(x^{\prime}\right)} g^{2}\left(x^{\prime}, x_{N}\right) d x_{N} \geqslant \int_{\Omega^{\star}} g^{2} d x
$$

this shows that $\Omega^{\star}$ is also admissible for the minimization problem (11). Denote by $u^{\star}$ the Steiner symmetrand of $u_{\Omega}$, that is the function whose level sets are the Steiner symmetrand of level sets of $u_{\Omega}$. By Fubini Theorem and classical results on rearrangement, it is well known that

$$
\begin{gathered}
\int_{\Omega} u_{\Omega} d x=\int_{\Omega^{\star}} u^{\star} d x, \\
\int_{\Omega}\left|\nabla u_{\Omega}\right|^{2} d x \geqslant \int_{\Omega^{\star}}\left|\nabla u^{\star}\right|^{2} d x .
\end{gathered}
$$

Thus, since $u^{\star}$ belongs to the Sobolev space $H_{0}^{1}\left(\Omega^{\star}\right)$, using the variational characterization (4) we get

$$
J\left(\Omega^{\star}\right)=G_{\Omega^{\star}}\left(u_{\Omega^{\star}}\right) \leqslant G_{\Omega^{\star}}\left(u^{\star}\right) \leqslant G_{\Omega}\left(u_{\Omega}\right)=J(\Omega),
$$

and the proof is complete.

\section{A relation with the level sets of $g$}

As largely proved in the previous sections, clearly the geometry of $g$ influences the geometry of the solution $\Omega$. Following the radial case, one could expect the shape of $\Omega$ to be strongly related to the shape of the level sets of $g$. Indeed in the radial case, $\Omega$ is an homothetic copy of the level sets of $g$; unfortunately this happens only in this particular case, as can be easily inferred from Serrin result [32]. On the other hand, some estimate of the solution $\Omega$ in terms of $g$ is always possible and this is precisely the aim of this final section. Roughly speaking, we will show that $\Omega$ must be incapsulated between two a priori known level sets of $g$. To give a precise statement, it is convenient to introduce first some notations.

As before, assume $g$ to be homogeneous of degree $\alpha>0$ and $g(x)>0$ if $x \neq 0$. Let $t \in(0, \infty)$ and denote by $G_{t}$ the (open) $t$-sublevel set of $g$, that is

$$
G_{t}=\left\{x \in \mathbb{R}^{n}: g(x)<t\right\} .
$$

By homogeneity, it is easily seen that all the level sets are dilatation of $G_{1}$, precisely

$$
G_{t}=t^{\frac{1}{\alpha}} G_{1} \text {. }
$$

Now let $u_{t}$ be the stress function of $G_{t}$ and assume that $g$ is regular enough to get $u_{t} \in C^{2}\left(G_{t}\right) \cap$ $C^{1}\left(\bar{G}_{t}\right)$ (for instance $g \in C^{1, \beta}\left(\mathbb{R}^{N}\right)$ for some $\beta>0$ ).

In particular $u_{1}$ is the solution of

$$
\begin{cases}-\Delta u_{1}=1 & \text { in } G_{1} \\ u_{1}=0 & \text { on } \partial G_{1}\end{cases}
$$

and it holds

$$
u_{t}(x)=t^{2 / \alpha} u_{1}\left(\frac{x}{t^{1 / \alpha}}\right)
$$




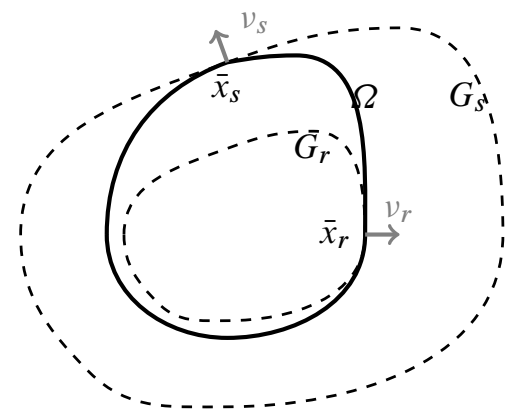

FIG. 5. $G_{r} \subseteq \Omega \subseteq G_{S}$

Set

$$
A=\min _{\partial G_{1}}\left|\nabla u_{1}\right|, \quad B=\max _{\partial G_{1}}\left|\nabla u_{1}\right| .
$$

Notice that $A$ and $B$ depends only on $g$ and they are, in principle, a priori known. Moreover, $A \leqslant B$ and $A<B$ unless $G_{1}$ is a ball (see [32]), that is $g$ is radial; the radial case is discussed in detail in the previous section.

THEOREM 7.1 If $\alpha>1$ and $\Omega$ satisfies (R) then

$$
A^{1 /(\alpha-1)} G_{1} \subseteq \Omega \subseteq B^{1 /(\alpha-1)} G_{1} .
$$

Proof. Since the origin $O$ is in the interior of both $\Omega$ and $G_{1}$, and they are both bounded, there exist $r$ and $s$ such that $0<r \leqslant s$ and

$$
r=\sup \left\{t: G_{t} \subseteq \Omega\right\} \quad \text { and } \quad s=\inf \left\{t: \Omega \subseteq G_{t}\right\} .
$$

Then $G_{r} \subseteq \Omega \subseteq G_{s}$ and there exist

$$
x_{r} \in \partial G_{r} \cap \partial \Omega \quad \text { and } \quad x_{s} \in \partial \Omega \cap \partial G_{s} .
$$

We want to estimate $r$ and $s$ in terms of $g$. Then let $w_{r}=u_{\Omega}-u_{r}$, where $u_{\Omega}$ is as usual the stress function of $\Omega$, and notice that $w_{r}$ satisfies

$$
\begin{cases}-\Delta w_{r}=0 & \text { in } G_{r} \\ w_{r} \geqslant 0 & \text { on } \partial G_{r} \\ w_{r}\left(x_{r}\right)=0 . & \end{cases}
$$

By maximum principle $w_{r} \geqslant 0$ in $G_{r}$ and then $x_{r}$ is a minimum point. Whence

$$
\frac{\partial w_{r}}{\partial v_{r}}\left(x_{r}\right)=\frac{\partial u_{\Omega}}{\partial v_{r}}\left(x_{r}\right)-\frac{\partial u_{r}}{\partial v_{r}}\left(x_{r}\right)=\left|\nabla u_{r}\left(x_{r}\right)\right|-\left|\nabla u_{\Omega}\left(x_{r}\right)\right| \leqslant 0,
$$

where $v_{r}$ is the outer normal of $G_{r}$ (and $\Omega$ ) at $x_{r}$. Since $\Omega$ solves Problem (2) and $x_{r} \in \partial G_{r}$, the latter reads

$$
\left|\nabla u_{r}\left(x_{r}\right)\right| \leqslant g\left(x_{r}\right)=r .
$$


On the other hand, (44) gives

$$
\left|\nabla u_{r}\left(x_{r}\right)\right|=r^{1 / \alpha}\left|\nabla u_{1}\left(\frac{x_{r}}{r^{1 / \alpha}}\right)\right|
$$

and thanks to (42) it holds

$$
\frac{x_{r}}{r^{1 / \alpha}} \in \partial G_{1} .
$$

Then from (45) we get

$$
r \geqslant r^{1 / \alpha} A,
$$

which implies, for $\alpha>1$,

$$
r \geqslant A^{\alpha /(\alpha-1)} ;
$$

and this proves the first inclusion of the statement, using (42).

To obtain the second inclusion we argue in a similar way. Let $w_{s}=u_{s}-u_{\Omega}$ and notice that it solves

$$
\begin{cases}-\Delta w_{s}=0 & \text { in } \Omega \\ w_{s} \geqslant 0 & \text { on } \partial \Omega \\ w_{s}\left(x_{s}\right)=0 & \end{cases}
$$

Arguing as before we get

$$
\left|\nabla u_{s}\left(x_{s}\right)\right| \geqslant g\left(x_{s}\right)=s .
$$

Coupling the latter with (44) and taking again into account (42) and (45), we get

$$
s \leqslant s^{1 / \alpha} B,
$$

whence, if $\alpha>1$, we obtain

$$
s \leqslant B^{\alpha /(\alpha-1)},
$$

which proves the second inclusion of the statement, using again (42).

Acknowledgements. The authors wish to warmly thank Dorin Bucur for several helpful discussions.

This paper started while Chiara Bianchini was supported by the project ANR-09-BLAN-0037 Geometric analysis of optimal shapes (GAOS) and by the research group INRIA Contrôle robuste infini-dimensionnel et applications (CORIDA).

The work of Antoine Henrot is part of the projects ANR-09-BLAN-0037 Geometric analysis of optimal shapes (GAOS) and ANR-12-BS01-0007-01-OPTIFORM Optimisation de formes financed by the French Agence Nationale de la Recherche (ANR).

The work of Chiara Bianchini and Paolo Salani is part of the projects GNAMPA 2012 Problemi sovradeterminati e geometria delle soluzioni per equazioni ellittiche e paraboliche and PRIN 2008 Aspetti geometrici delle equazioni alle derivate parziali e questioni connesse. 


\section{REFERENCES}

1. ACKer, A., Interior free boundary problems for the Laplace equation, Arch. Rational Mech. Anal. 75 (1980/81), 157-168. Zbl0397.76009 MR0605527

2. ACKER, A., Uniqueness and monotonicity of solutions for the interior Bernoulli free boundary problem in the convex $n$-dimensional case, Nonlinear Anal. 13 (1989), n. 12, 1409-1425. Zb10714.35085 MR1028238

3. Aftalion, A., Busca, J. \& Reichel, W., Approximate radial symmetry for overdetermined boundary value problems, Adv. Diff. Eq. 4 (1999), 907-932. Zbl0951. 35046 MR1729395

4. Agostiniani, V. \& Magnanini, R., Symmetries in an overdetermined problem for the Green's function. Discrete Contin. Dyn. Syst. Ser. S 4 (2011), 791-800. Zbl1210. 35169 MR2746441

5. Alt, H. W. \& CAfFARElli, L.A., Existence and regularity for a minimum problem with free boundary, J. Reine Angew. Math., 325 (1981), 105-144. Zb10449. 35105 MR0618549

6. Bianchini, C., A Bernoulli problem with non constant gradient boundary constraint, Appl. Anal. 91 (2012), 517-527. Zbl1238.35192 MR2876741

7. Brandolini, B., Nitsch, C., Salani, P. \& Trombetti, C., On the stability of the Serrin problem, J. Diff. Equations 245, Issue 6 (2008), 1566-1583. Zbl1173. 35019 MR2436453

8. BRIANÇON, T., Regularity of optimal shapes for the Dirichlet's energy with volume constraint, ESAIM: COCV, 10 (2004), 99-122. Zbl1118. 35078 MR2084257

9. Briançon, T., Hayouni, M. \& Pierre, M., Lipschitz Continuity of State Functions in Some Optimal Shaping, Calculus of Variations and PDE's 23 (2005), 13-32. Zbl1062 . 49035 MR2133659

10. Bucur, D., Uniform Concentration-Compactness for Sobolev spaces on variable domains, J. Diff. Eq. 162 (2000), 427-450. Zb10957.49027 MR1751712

11. Bucur, D., Minimization of the $k$-th eigenvalue of the Dirichlet Laplacian, Arch. Rational Mech. Anal. 206-3 (2012), 1073-1083. Zbl1254.35165 MR2989451

12. Bucur, D., Buttazzo, G. \& Velichkov, B., Shape Optimization Problems with Internal Constraint, preprint arXiv:1109.2413.

13. Caffarelli, L. A. \& Spruck, J., Convexity properties of solutions to some classical variational problems, Comm. Partial Differential Equations 7 (1982), 1337-1379. Zb10508. 49013 MR0678504

14. Choe, J., Ghomi, M. \& Ritoré, M., The relative isoperimetric inequality outside convex domains in $R^{n}$. Calc. Var. Partial Differential Equations 29 (2007), 421-429. Zb11116.58016 MR2329803

15. Cianchi, A. \& S Alani, P., Overdetermined anisotropic elliptic problems, Math. Ann. 345 (2009), 859881. Zbl1179.35107 MR2545870

16. Ciraolo, G., Magnanini, R. \& Sakaguchi, S., Symmetry of minimizers with a level surface parallel to the boundary, To appear in J. Eur. Math. Soc.

17. Colesanti, A. \& SAlani, P., Quasi-concave envelope of a function and convexity of level sets of solutions to elliptic equations, Math. Nachr. 258 (2003), 3-15. Zbl1128. 35332 MR2000041

18. CrouzeiX, M., Variational approach of a magnetic shaping problem, Eur. J. Mech. B Fluids 10 (1991), 527-536. Zbl0741.76089 MR1139607

19. Gustafsson, B. \& Shahgholian, H., Existence and geometric properties of solutions of a free boundary problem in potential theory, J. Reine Angew. Math. 473 (1996), 137-179. Zb10846. 31005 MR1390686

20. Greco, A., Symmetry around the origin for some overdetermined problems, Adv. Math. Sci. Appl. 13 (2003), 387-399. Zbl1078.35037 MR2002730

21. Greco, A., A characterization of the ellipsoid through the torsion problem, Z. Angew. Math. Phys. 59 (2008), 753-765. Zbl1160.35385 MR2442948

22. Henrot, A. \& Philippin, G. A., Some overdetermined boundary value problems with elliptical free boundaries, SIAM J. Math. Anal. 29 (1998), 309-320. Zb10921.35196 MR1616562 
23. Henrot, A. \& Pierre, M., Variation et Optimisation de forme, une analyse géométrique, Mathématiques et Applications, 48, Springer 2005. Zbl1098.49001 MR2512810

24. Henrot, A \& Shahgholian, H., The one phase free boundary problem for the $p$-Laplacian with nonconstant Bernoulli boundary condition, Trans. Amer. Math. Soc. 354 (2002), 2399-2416. Zb10988. 35174 MR1885658

25. Kawohl, B., Rearrangements and Convexity of Level Sets in P.D.E., Lecture Notes in Mathematics, 1150, Springer, Berlin, 1985. Zb10593.35002 MR0810619

26. Kennington, A. U., Power concavity and boundary value problems, Indiana Univ. Math J. 34 (1985), 687-704. Zbl0549.35025 MR0794582

27. KorevaAr, N. J. Convex solutions to nonlinear elliptic and parabolic boundary value problems, Indiana Univ. Math. J. 32 (1983), 603-614. Zbl0481. 35024 MR0703287

28. Morrey, C. B., Multiple integrals in the calculus of variations, Springer 1966. Zbl0142.38701 MR0202511

29. Pólya, G., Torsional rigidity, principal frequency,electrostatic capacity and symmetrization, Quart. Appl. Math. 6 (1948), 267-277. Zbl0037.25301 MR0026817

30. Pólya, G. \& Szegö, G., Isoperimetric inequalities in mathematical physics, Ann. Math. Studies 27, Princeton Univ. Press, 1951. Zbl044.38301 MR0043486

31. SAlani, P., Characterization of balls through optimal concavity for potential functions, To appear in Proc. AMS.

32. SERrin, J., A symmetry problem in potential theory, Arch. Rational Mech. Anal. 43 (1971), 304-318. Zb10222.31007 MR0333220

33. Schaefer, P. W., On nonstandard overdetermined boundary value problems, Proceedings of the Third World Congress of Nonlinear Analysis, Part 4 (Catania, 2000). Nonlinear Anal. 47 (2001), 2203-2212. Zbl1042.35530 MR1971630

34. Shahgholian, H., Diversifications of Serrin's and related problems, Complex Var. and Elliptic Eq. 57 (2012), 653-665. Zbl1269.35021 MR2916825 